\title{
Benefits of Tai Chi for fibromyalgia
}

\author{
Jana Sawynok*,1 \\ ${ }^{1}$ Departments of Pharmacology, Anesthesia, Perioperative Medicine \& Pain Management, Dalhousie University, Halifax, Nova \\ Scotia, Canada \\ *Author for correspondence: jana.sawynok@dal.ca
}

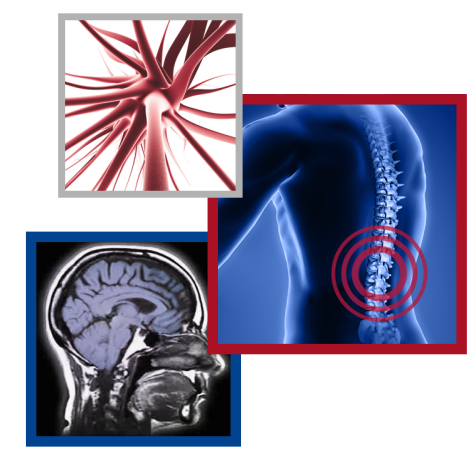

\section{"Tai Chi involves 'something else' beyond physical movement and enhancing fitness"}

First draft submitted: 1 May 2018; Accepted for publication: 4 May 2018; Published online: 5 June 2018

Keywords • complementary therapy • fibromyalgia • meditative movement therapy • Tai Chi

Fibromyalgia affects approximately $2 \%$ of general populations - it is a difficult condition to experience for patients, a challenging condition to treat for clinicians and a costly condition to address for societies. Conceptualization of fibromyalgia has evolved over the decades since it was recognized as a clinical entity in the early 1990s by the American College of Rheumatology, with reformulation in 2010 emphasizing the chronic and widespread nature of the pain, symptom severity and the presence of comorbidities [1,2]. In 2016, a white paper detailing challenges in the field was published to provide a framework for global efforts to improve understanding and treatment of fibromyalgia [3]. Current recommendations for management of fibromyalgia stratify approaches into pharmacological therapies and complementary and alternative medicine approaches [4]. These include patient education, physical therapy and graded exercise, together with individualized treatments depending on the impact of the condition on function. Challenges to implementing these guidelines include recognizing that fibromyalgia diagnosis can take multiple years and patients will often already be on several drugs for pain and comorbidities, the role of physical therapy/exercise before or along with pharmacotherapy has generally not been evaluated, along with challenges of exercise regimens in this particular population [5]. This overview acknowledges that several forms of complementary and alternative medicines show promise but require rigorous testing.

Tai Chi has a long history in China as a health and wellness martial arts practice [6] and has been designated a 'complex intervention' [7]. It involves core elements of specific movements, meditative instruction and breath regulation, and more recently has been characterized as a 'meditative movement' to provide contemporary understanding and to distinguish it from other exercises and meditative regimens [8]. It is also considered as a 'complementary' or 'mindful' exercise and is clustered along with practices such as yoga, meditation and mind-body practices in overview analyses. Research into health benefits of Tai Chi has been steadily growing since the late 1990s, with 100-200 publications/year appearing between 2010-2015, and currently there is considerable interest in this subject [9]. A 2016 meta-analysis of Tai Chi for several chronic pain conditions provides support for its usefulness for osteoarthritis, low back pain and osteoporosis [10].

Between 2010 and 2017, five studies involving 36-100 subjects with fibromyalgia, reported benefits (compared with control groups or before/after comparisons) of Tai Chi in core symptom domains for this condition (pain, sleep, impact, physical function and mental function) [11-15]. These studies involved supervised Tai Chi instruction and practice sessions two to three times per week for 60-90 min (one involved additional daily home practice for $20 \mathrm{~min}$ ), lasted 12-28 weeks and generally used modified 8-10 Form Yang Style Tai Chi (one involved Tai Ji Quan). While these studies uniformly reported health benefits and are promising, the heterogeneity of study designs, particularly with respect to duration of supervised practice and the presence or absence of home practice, confounds clear interpretation, especially as benefits of another meditative movement practice (qigong) in fibromyalgia are related to amount of practice [16].

In 2018, a comparative trial, involving 226 participants with fibromyalgia, compared effectiveness of Tai Chi with aerobic exercise [17]. This is an important study because it compared Tai Chi against the most commonly prescribed nondrug treatment (aerobic exercise); it used well-validated measures (primary outcome measure of Fibromyalgia Impact Questionnaire Revised, a multi-dimensional measure of pain, physical function, fatigue, morning tiredness, depression, anxiety, job difficulty and overall well being, there were several additional secondary 
outcomes); it assessed two regimens of Tai Chi (12 or 24 weeks, once or twice weekly) and included recommended home practice of $30 \mathrm{~min}$ daily; it involved long-term follow-ups to 52 weeks. The main results are as follows: compared to baselines, Fibromyalgia Impact Questionnaire Revised scores in all five groups (Tai Chi $1 \times 12$ weeks, $2 \times 12$ weeks, $1 \times 24$ weeks, $2 \times 24$ weeks and aerobic exercise $2 \times 24$ weeks) were improved; benefits in the combined Tai Chi groups were significantly greater than in the aerobic exercise group in Fibromyalgia Impact Questionnaire Revised scores and in several secondary outcomes; benefits in the 24-week Tai Chi group compared with the matched intensity and duration of aerobic exercise group showed significantly greater benefits in the Tai Chi group; there were only a few differences between 12 and 24-week Tai Chi groups. Of note, participants in all groups decreased their analgesic medication use by the end of the study, providing a further indication of improvements in pain.

In summary, the 2018 study reports that both aerobic exercise and Tai Chi produce multiple health benefits in fibromyalgia subjects who have associated comorbidities and a poor quality of life, and results are of considerable clinical and public health relevance. Baseline characteristics indicated a mean pain duration of about a decade in each group and multiple pharmacological regimens; results indicate further benefits with Tai Chi and exercise, and so are of importance for the design of multi-modal treatment programs. Of particular note, with the greater benefits observed in the Tai Chi group compared with the matched aerobic exercise group, it appears that Tai Chi involves 'something else' beyond physical movement and enhancing fitness. From a theoretical perspective, given the emerging literature on health benefits of Tai Chi in many diverse health areas [9], there is an intrinsic appeal to a practice that can potentially provide clinical benefits in multiple areas. Along these lines, a recent comparative study of physical therapy versus Tai Chi for knee osteoarthritis reported comparable effectiveness in arthritis measures, but greater improvements in depression and physical quality of life in the Tai Chi group [18]. There is a considerable scope for assessing cost-effectiveness of interventions of this nature that can be administered in group settings.

Several further aspects of the 2018 trial warrant consideration, and these have implications for future studies. Firstly, there were attrition rates of $11-28 \%$ at 24 weeks and $25-35 \%$ at 52 weeks. These are not unusual given the requirements and duration of the trial and the patient population. A consequence of attrition is the need to rely on imputation models (efficacy analysis), each of which has attendant assumptions. A real-world pragmatic perspective recognizes that particular practices may not be for everyone, and considers that effects of a particular practice can only be determined in those who actually engage the practice. The study comments on the robustness of observations even with various imputation models, but a real-world per protocol analysis would also be beneficial. Future studies will need to address which patients remain in studies and attain the best outcomes. Exploration can include general attributes such as expectations (which contribute to placebo), health locus of control (which contributes to self-efficacy), and attitudes towards complementary and alternative medicine (which contribute to treatment preferences). It could also include monitoring specific features that might be particularly relevant to Tai Chi such as measures of mindfulness and of body awareness/interoception (several have been developed in these domains) or measures specific for Tai Chi practice (e.g., meditative movement inventory).

Secondly, the study monitors adherence to attendance at supervised sessions, but does not record daily practice using self-reported logs. While there are limits to the latter, they can provide an additional indication of adherence. The study notes that attendance at Tai Chi sessions is greater than attendance at exercise sessions. Going forward, it will be important to determine which methods optimize engagement and adherence and to include these in the study design. The current study emphasized equipoise as an element of trial design in order to minimize bias, which is a laudable feature. However, as health benefits of Tai Chi reveal themselves through further studies, especially with attention to the issue of amount of practice, there may need to be additional ways of framing information to encourage and enhance adherence. Meta-analysis approaches will also need to recognize the issue of amount of practice, and stratify their analysis of study outcomes in relation to this factor. At present, while there is often some mention of this as a potential factor of importance, there has been little systematic attention directed at the issue.

A final challenge for the future is to understand mechanisms by which Tai Chi produces health benefits. Within a traditional framework, it involves cultivation of qi (chi) and promotion of a free and unhindered flow of energy. Contemporary viewpoints consider it as a complex intervention integrating concepts of mind and body, with physical, emotional and spiritual aspects, or use specific terminology to distinguish it from other forms of movement or static meditative practices $[7,8]$. Emerging aspects of focus include assessments of effects on autonomic function providing a monitor of bodily function [19] and brain imaging and network analysis which reflects neural and integrative dimensions [20]. Tai Chi potentially recruits integrative elements of systems biology that are now being articulated, and going forward, empirical studies of clinical outcomes that consider subgroups (an aspect of 
more personalized medicine), as well as exploratory studies of mechanisms, will be required to better recruit the modern clinical potential of this ancient practice.

Financial \& competing interests disclosure

The author has no relevant affiliations or financial involvement with any organization or entity with a financial interest in or financial conflict with the subject matter or materials discussed in the manuscript. This includes employment, consultancies, honoraria, stock ownership or options, expert testimony, grants or patents received or pending, or royalties.

No writing assistance was utilized in the production of this manuscript.

\section{References}

Papers of special note have been highlighted as: • of interest; $\bullet \bullet$ of considerable interest

1 McBeth J, Mulvey MR. Fibromyalgia: mechanisms and potential impact of the ACR 2010 classification criteria. Nat. Rev. Rheumatol. $8(2), 108-116$ (2012).

2 Fitzcharles MA, Ste-Marie P, Pereira JX. Fibromyalgia: evolving concepts over the past 2 decades. CMAJ 185(13), E645-E651 (2013).

3 Arnold LM, Choy E, Clauw DJ et al. Fibromyalgia and chronic pain syndromes. A white paper detailing current challenges in the field. Clin. J. Pain. 32(9), 737-746 (2016).

4 MacFarlane GJ, Kronisch C, Dean LE et al. EULAR revised recommendations for the management of fibromyalgia. Ann. Rheum. Dis. 76(2), 318-328 (2017).

- Updated recommendations on management strategies for fibromyalgia. Includes a separate consideration of pharmacological and complementary and alternative medicine strategies. Identifies aerobic exercise as a core recommendation for all patients with fibromyalgia.

5 Arnold LM, Clauw DJ. Challenges of implementing fibromyalgia treatment guidelines in current clinical practice. Postgrad. Med. 129(7), 709-714 (2017).

6 Jahnke R. The healing promise of Qi. Creating extraordinary wellness through qigong and Tai Chi. McGraw Hill, NY, USA (2002).

7 Wayne PM, Kaptchuk TJ. Challenges inherent to T'ai Chi research: part I - T'ai Chi as a complex multicomponent intervention. J. Altern. Complement. Med. 14(1), 95-102 (2008).

8 Payne P, Crane-Godreau MA. Meditative movement for depression and anxiety. Front. Psychiatry 4, 71 (2013).

9 Huston P, McFarlane B. Health benefits of Tai Chi. What is the evidence? Can. Fam. Phys. 62(NOV), 881-890 (2016).

- Presents a publication timeline for Tai Chi studies which indicates particular growth since the late 1990s. It summarizes evidence into areas where there is excellent, good, fair, preliminary or no evidence of benefit. However, the focus is on the information in systematic reviews and there is no consideration of adherence or practice time.

10 Kong LJ, Lauche R, Klose P et al. Tai Chi for chronic pain conditions: a systematic review and meta-analysis of randomized controlled trials. Sci. Rep. 6, 25325 (2016).

-• Provides a meta-analysis of Tai Chi for several chronic pain conditions. Effect sizes, or standard mean difference, are - 0.54 for osteoarthritis, $\mathbf{- 0 . 8 1}$ for low back pain and $\mathbf{- 0 . 8 3}$ for osteoporosis. It concludes that Tai Chi is a viable approach for chronic pain conditions.

11 Wang C, Schmidt CH, Rones R et al. A randomized trial of Tai Chi for fibromyalgia. New Engl. J. Med. 363(8), 743-754 (2010).

- Comparative trial of Tai Chi with a control group involving education and stretching. Tai Chi provided clinically important improvements in multiple outcomes and was superior to the control group. This study provided information on adequacy of the regimen for power analysis and informed the 2018 study [17].

12 Romero-Zurita A, Carbonell-Baeza A, Aparicio VA et al. Effectiveness of a Tai-Chi training and detraining on functional capacity, symptomology and psychological outcomes in women with fibromyalgia. Evid. Based Complement. Alternat. Med. 2012, $614186 \mathrm{~m}(2012)$.

13 Jones KD, Sherman CA, Mist SD et al. A randomized controlled trial of 8-form Tai Chi improves symptoms and functional mobility in fibromyalgia patients. Clin. Rheumatol. 31(8), 1205-1214 (2012).

14 Segura-Jiménez V, Romero-Zurita A, Carbonell-Baeza A et al. Effectiveness of Tai-Chi for decreasing acute pain in fibromyalgia patients. Int. J. Sports Med. 35(5), 418-423 (2014).

15 Maddali Bongi S, Paoletti G, Calà M et al. Efficacy of rehabilitation with Tai Ji Quan in an Italian cohort of patients with fibromyalgia syndrome. Complement. Ther. Clin. Pract. 24, 109-115 (2016).

16 Lynch M, Sawynok J, Hiew C et al. A randomized controlled trial of qigong for fibromyalgia. Arthritis Res. Ther. 14(4), R178 (2012).

17 Wang C, Schmid CH, Fielding RA et al. Effect of Tai Chi versus aerobic exercise for fibromyalgia: comparative effectiveness randomized controlled trial. BMJ 360, k851 (2018).

-. The main article featured in this editorial. The study has many notable features in terms of design, implementation and analysis, and meets rigorous standards in trial design and reporting. This study has important clinical and public health implications. 
18 Wang C, Schmid CH, Iversen MD et al. Comparative effectiveness of Tai Chi versus physical therapy for knee osteoarthritis. Ann. Intern. Med. 165(2), 77-86 (2016).

-. Comparative trial involving Tai Chi versus physical therapy. Arthritis outcomes (WOMAC) were similar between groups, but Tai Chi had better outcomes on mental and physical health. Given the ability to deliver Tai Chi instruction in groups, cost-effectiveness analysis is warranted.

19 Wong A, Figueroa A, Sanchez-Gonzalez et al. Effectiveness of Tai Chi on cardiac autonomic function and symptomology in women with fibromyalgia: a randomized controlled trial. J. Aging Phys. Act. 23, 1-8 (2018).

20 Yu AP, Tam BT, Lai CW et al. Revealing the neural mechanisms underlying the beneficial effects of Tai Chi: a neuroimaging perspective. Am. J. Chin. Med. 46(2), 231-259 (2018). 